Koedoe 16: 155-158 (1973).

\title{
A RETRACTABLE BARB NEEDLE FOR DRUG DARTS
}

\author{
by \\ G. L. VAN ROOYEN* and P. J. DE BEER*
}

\begin{abstract}
The mechanism and action of a new retractable barb needle for drug darts are described. This dart needle is particularly successful in obviating unnecessary flight reactions and trauma in darted animals, and facilitates the complete injection of the drug dose before the barb is retracted and the dart is dislogded from the animal. The whole process is completed within a few seconds and the expended dart can usually be retrieved in the immediate vicinity where the animal was darted.
\end{abstract}

\section{Introduction}

Experience in the field by the research staff of the Kruger National Park has shown that when a dart is fired and retained in an animal by a device such as a barb or conical enlargement of the needle, the darted animal will be stimulated to run long distances. This may result from irritation caused by the dart, and because it is frightened by the sight of the dart. This sustained flight reaction often results in the development of capture myopathy reactions, which when severe are almost invariably fatal. In this process the dart can also be lost or damaged by the animal trying to rid itself of the dart by brushing against trees and shrubs, or by falling on the dart when the drug takes effect.

Experience has shown that if an animal is darted with a small capacity dart $(0,5-1,0 \mathrm{ml})$ delivering the full volume of the drug on impact and with no retaining device used on the needle, such animals, without exception, did not run more than a short distance and the darts were recovered intact.

Where the volume of drugs to be administered exceeded 1,0 ml, however, this method of using barbless needles cannot be used. This led to the development by one of us (P. J. de B.) of the retractable barb needle. This innovation will allow the barb to fall out only after the total volume of drug has been delivered.

\section{* Kruger National Park, P.O. Box X404, Skukuza.}




\section{Description}

Figure 1 shows the three component parts of this needle, comprising:

(i) the dart head with the needle attached, a slot machined in the needle near the sharp end, and a small hole drilled in close proximity to, and in line with the slot, between the latter and the dart head;

(ii) the barb itself consisting of a spring steel wire, one end of which is bent in the shape of a long-stemmed " $\delta$ " as shown, and with a metallic disc of diameter slightly less than the bore of the dart barrel, welded on the other end; and

(iii) a conical spring.

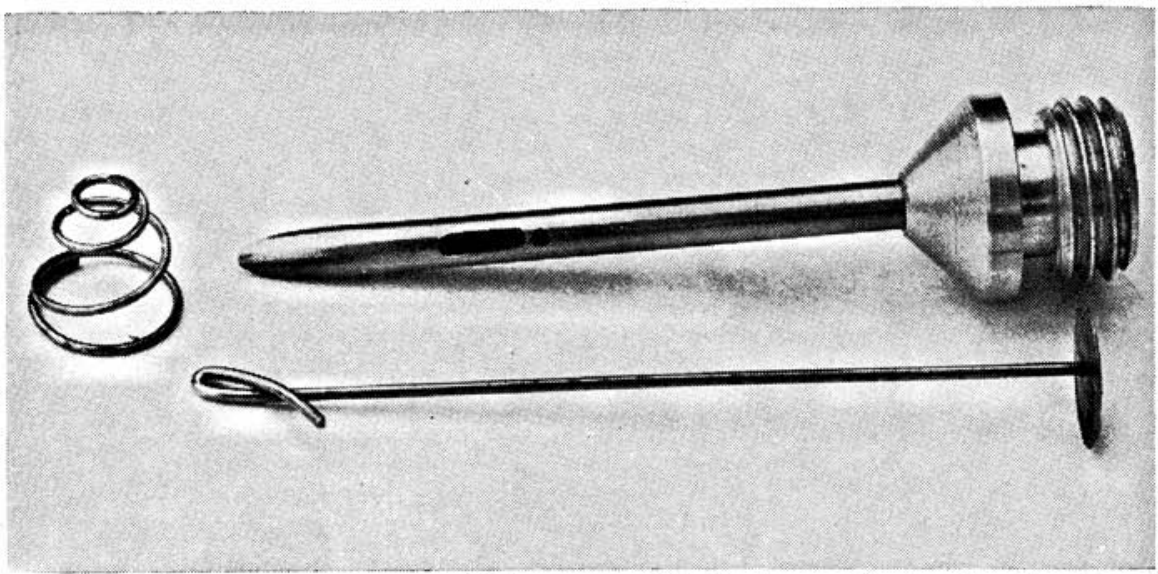

Fig. 1. The component parts of the retractable barb needle, i.e. dart head and needle, spring steel barb, and conical spring, are shown. Note the slot and hole in the needle near the sharpened end, the shape of the barb and the disc welded on the other end.

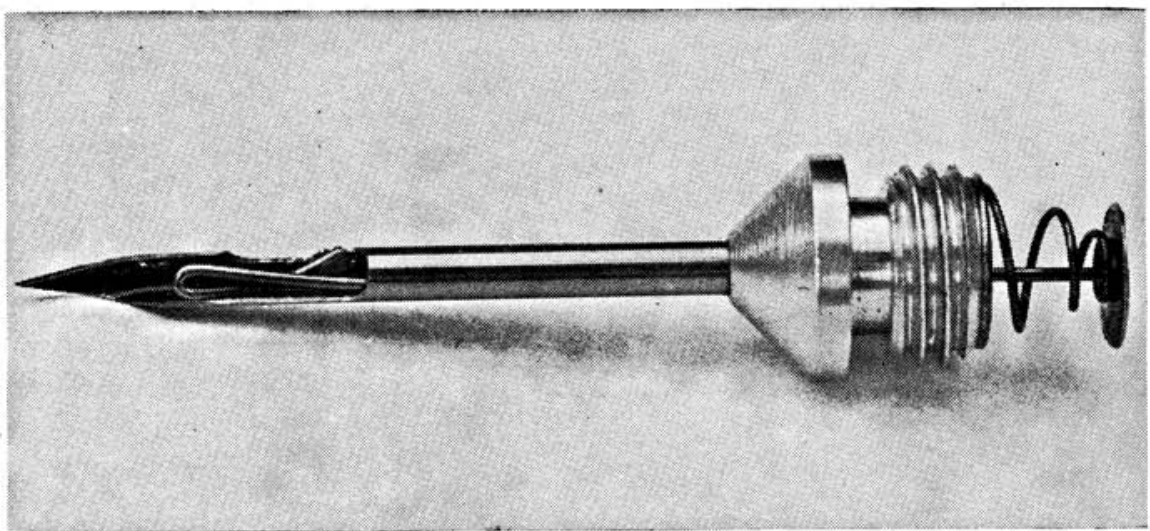

Fig. 2. A partially sectioned needle showing the barb in the "cocked" position. Note the tip of the compressed barb "anchored" in the hole. Rearward movement of the barb relative to the needle is thereby prevented. 


\section{Assembly}

The cone spring is slipped onto the barb so that the small end is in contact with the disc, and the " "-shaped end of the barb pushed into the needle from the threaded end of the dart head until the tip of the barb, which is compressed inside the needle, clicks into the small hole. Figure 2 shows the barb in this "cocked" position. Assembling the needle should be seen as part of the process of loading the dart.

\section{Operation}

- The needle with the barb in the "cocked" position when screwed onto a loaded drug dart, is ready to be fired into an animal. The barb, being compressed inside the bore of the needle and not protruding from it,

1 cannot be damaged when piercing the hide, and being anchored in the hole will not become dislodged when the dart accelerates upon being projected.

On impact with the target, the barb, by virtue of its own inertia, and the impact of the drug on the disc, is dislodged from the hole (the shape of the barb facilitating this action), moves forward and permits the spring " steel barb to snap outwards through the slot into the "working" position, as shown in Fig. 3. Undue forward movement of the barb is checked by the spring, which will also hold the barb in the "working" position, thus retaining the dart in the animal.

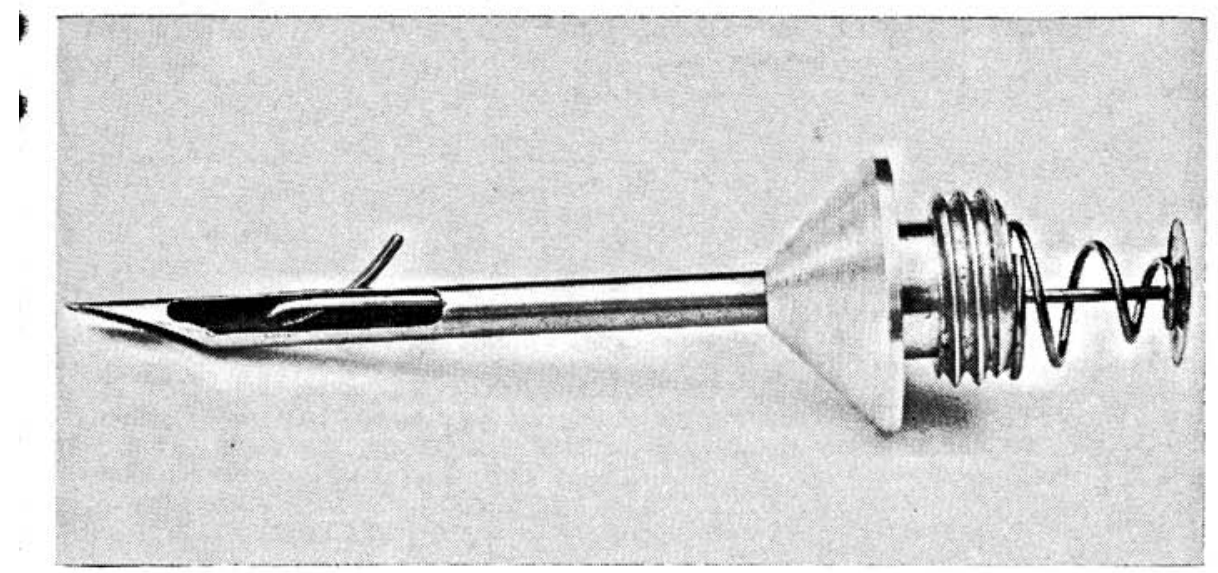

Fig. 3. The barb shown in the extended or "working" position.

As the dart plunger moves forward in the process of injecting the drug, it comes into contact with the disc towards the end of its delivery stroke, and pushes the barb forward until it makes contact with the distal end of the slot. Further pressure compresses the barb into the needle again and it is completely retracted by the time the cone spring is fully compressed by the plunger, as shown in Fig. 4. The dart is now no longer retained mechanically in the animal and is free to fall out. 


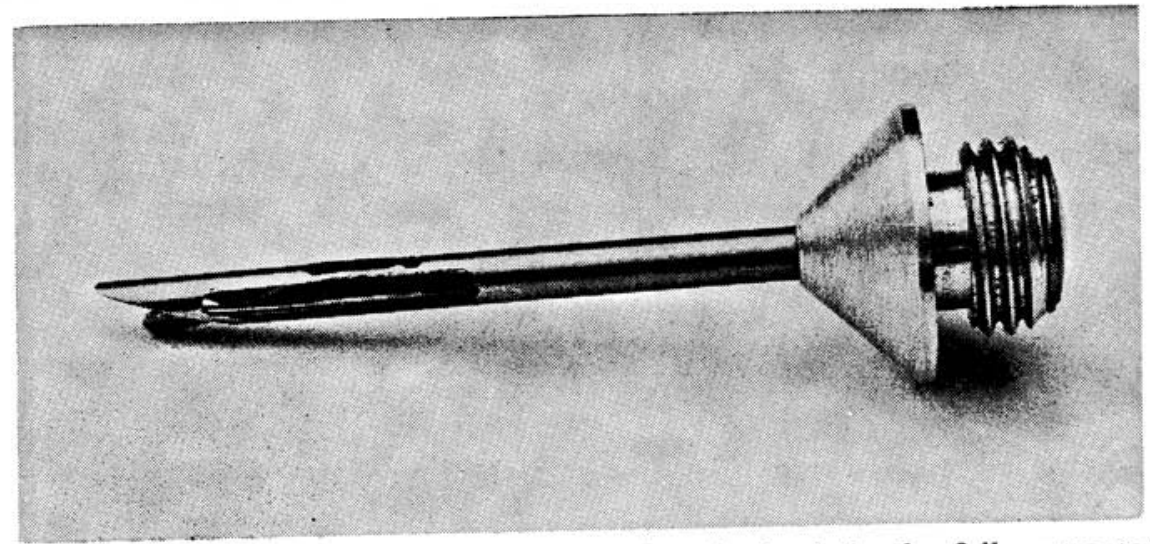

Fig. 4. A partially sectioned needle showing the barb in the fully retracted position with the conical spring fully compressed.

This operation obviously depends on sufficient plunger pressure and the device can only be used in darts where the pressure is adequate, such as those in which plunger movement is facilitated by a mixture of bicarbonate and acid. The whole process is completed within a few seconds and the expended dart can usually be retrieved in the immediate vicinity where the animal was darted.

\section{Maintenance}

Fully compressing the cone spring by hand to get the barb into the retracted position and twisting the barb clockwise (viewed from the rear) about one eighth turn to clear the slot will enable the barb to be pulled out of the needle for cleaning. To reassemble, instructions under "assembly" (above) should be followed. 


\title{
XYLAZINE HYDROCHLORIDE (ROMPUN) AND THE NEW RETRACTABLE-BARBED DART ("DROP-OUT" DART) FOR THE CAPTURE OF SOME NERVOUS AND AGGRESSIVE ANTELOPE SPECIES
}

\section{G. L. SMUTS*}

\begin{abstract}
The successful capture and restraint of gemsbok, eland and kudu using the potent sedative, Xylazine hydrochloride (Rompun, Bayer) and the new retractable-barbed dart ("dropout" dart) is described. These species were rapidly immobilized and rendered 100 per cent tractable by Etorphine/Rompun or Fentanyl/Rompun drug combinations. The drop-out dart proved to be invaluable for the capture of timid species as flight distances were greatly reduced and the chances of capture myopathy (overstraining disease) subsequently obviated.
\end{abstract}

\section{Introduction}

During the past decade very successful drug combinations and capture techniques have been developed for the restraint of most African ungulate

1 species. Some of these are described by Harthoorn (1963, 1965 and 1966), King and Carter (1965), Pienaar, Van Niekerk, Young, Van Wyk and Fairall (1966), Pienaar (1968 a and b), Pienaar (1969 and in press) and Hanks (1967). In a number of species there are, however, capture problems yet to be overcome or techniques that require improvement. When reviewing the literature it is obvious that most major problems are confined to the nervous and aggressive antelope species.

These species are usually difficult to approach with the capture vehicle (i.e. very timid), inclined to run long distances if the dart remains lodged in their bodies, and difficult to tranquillize.

Considering these problems and using a newly designed retractablebarbed projectile syringe ("drop-out" dart)** together with a drug mixture containing the potent sedative, Rompun (Bayer) in combination with Etorphine hydrochloride (Reckitt) or Fentanyl (Janssen) the following species were successfully captured:

\footnotetext{
* Biological Section, P.O. Box X404, Skukuza.

** (See paper by Van Rooyen and De Beer in this issue - Eds.)
} 